\title{
Kalman Filter Tracking
}

\author{
Nasser H. Ali \\ Ministry of Electricity \\ Department of Communication \\ Baghdad, Iraq
}

\author{
Ghassan M. Hassan \\ Al-Mustansiriyah University \\ Computer Department \\ Baghdad, Iraq
}

\begin{abstract}
Kalman filter estimates the state of a dynamic system, even if the precise form of the system is unknown. The filter is very powerful in the sense that it supports estimations of past and even future states. The description of the standard Kalman filter and its algorithms with the two main steps, the prediction step and the correction step. Furthermore the extended Kalman filter is discussed, which represents the conversion of the Kalman filter to nonlinear systems. Finally these filter was tested on aircraft tracking, and sinus wave using MATLAB.
\end{abstract}

\section{Keywords}

KF, EKF, Prediction, dynamic model, state vector

\section{INTRODUCTION}

Navigation system becomes standard, practically in aerospace, marine, demographic modeling, weather science manufacturing, new cars and many others[4]. Most of these navigation systems use no longer only the global positioning system (GPS) but also an inertial navigation system (INS) to help for finding the true way. Together the two systems complement each other and permit improved navigation accuracy and reliability especially when GPS is degraded or interrupted because of buildings or tunnels[4,5,6]. And for this application the kalman filter provides the basis. It constitutes a tool for correcting the predicted INS trajectory with GPS measurements.

\section{KALMAN FILTER}

The kalman filter is a recursive predictive filter that is based on the use of state space techniques and recursive algorithms[7]. It estimates the state of dynamic system. This dynamic system can be disturbed by some noise, mostly assumed as white noise. To improve the estimated state the kalman filter uses measurements that are related to the state but disturbed as well. Thus the kalman filter consists of two steps[7];

1-The prediction state, which predict with the dynamic model

2-The correction step, which is corrected with the observation model, so that the error covariance of the estimator is minimized it as an optimal estimator[7].

\section{STATE VECTOR}

The state vector contains the variables of interest (such as position, velocity, orientation angle, ....etc.). It describes the state of dynamic system and represent its degrees of freedom. The variables in the state vector cannot be measured directly, but they can be inferred from values that are measurable.

\section{DYNAMIC MODEL}

The dynamic model describes the transformation of the state vector with respect to time. It can usually be represented by system differential equation;

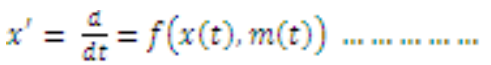

and in the linear case;

$x^{0}=F_{x} x(t)+n(t)$

Where $\mathrm{F}$ is the dynamic matrix and it is constant, $\mathrm{n}(\mathrm{t})$ is the dynamic noise with covariance matrix $\mathrm{Q}(\mathrm{t})[6,8]$.

\section{OBSRVATION MODEL}

The observation model represents the relationship between the state and the measurements. In the linear case, the measurements can be described by a system linear equations, which depend on the state variable;

$\mathbb{R}\left(t_{i}\right)=h\left(x\left(t_{i}\right), v\left(t_{i}\right)\right)$

So the vector form of this system is

$l\left(t_{i}\right)=H \cdot x\left(t_{i}\right)+w\left(t_{i}\right)$

Where $1\left(t_{i}\right)$ is the vector of the observation at the epoch $t_{i}, H$ is the observation matrix, and $\mathrm{w}\left(\mathrm{t}_{\mathrm{i}}\right)$ is the noise of the measurement process with the covariance matrix $R\left(t_{i}\right)$. Like the dynamic matrix, in a linear system, the observation matrix $\mathrm{H}$ is a constant matrix.

\section{KALMAN FILTER PREDICTION}

Prediction is the first step of kalman filter. The predicted state is calculated by neglecting the dynamic noise, and solving equation 2 . The covariance matrix $\mathrm{P}\left(\mathrm{t}_{\mathrm{i}}\right)$ of the predicted state vector is obtained with the law of error propagation;

$P^{-}\left(t_{i}\right)=\phi_{t_{i-1}}^{t_{i}} P\left(t_{i-1}\right) \cdot\left(\phi_{t_{i-1}}^{t_{i}}\right)^{T}+\int_{t_{i-1}}^{t_{i}} Q(t) d t \ldots \ldots(5)$

Where $\varnothing$ is called the state transition matrix, which transform any initial state $\mathrm{x}\left(\mathrm{t}_{\mathrm{o}}\right)$ to its corresponding state $\mathrm{x}(\mathrm{t})$ at time $\mathrm{t}$. Also the covariance matrix of noise $Q$ is a function of time $[9,10]$.

\section{KALMAN FILTEER CORRECTION}

In the correction step, the predicted state vector $\mathrm{x}^{-}\left(\mathrm{t}_{\mathrm{j}}\right)$ is improved with observations made at the epoch $t_{i}$, thus the posteriori state has the form. 
$x^{+}\left(t_{i}\right)=x^{-}\left(t_{i}\right)+\Delta x\left(t_{i}\right)$

With the covariance matrix $P^{+}\left(t_{i}\right)=P^{-}\left(t_{i}\right)+\Delta P\left(t_{i}\right)$

As a kalman filter is an optimal filter, this means that the state variances in the state covariance matrix $\mathrm{P}^{+}$are minimized. From the prediction step, $\mathrm{P}^{-}$is already known, it follows that $\Delta \mathrm{P}$ is minimized.

$$
\Delta P\left(t_{i}\right)=E\left[\Delta x\left(t_{i}\right) \Delta x\left(t_{i}\right)^{\mathbb{T}}\right]
$$

The corrected state is obtained by

$x^{+}\left(t_{i}\right)=x^{-\left(t_{i}\right)}+K\left(t_{i}\right) \cdot\left[\mathbb{l}\left(t_{i}\right)-\mathbb{l}^{-}\left(t_{i}\right)\right]$

Where $\mathrm{K}$ is called the gain matrix. The difference term is called the measurement residual. It reflects the discrepancy between the predicted measurement $\mathbb{I}^{-}\left(t_{i}\right)=\mathrm{Hx}^{+}\left(\mathrm{t}_{i}\right)$ and the actual measurement $l\left(t_{i}\right)$.

The covariance matrix of the a posteriori state is given with the law of error propagation.

$P^{+}\left(t_{i}\right)=P^{-}\left(t_{i}\right)-K\left(t_{i}\right) H P^{-}\left(t_{i}\right)$

\section{EXTENDED KALMAN FILTER}

This type of filter is used for nonlinear model. The disadvantage of this model is to need more time-consuming calculations. The implementation for linear systems can be made more efficient by pre-computing the dynamic matrix $\mathrm{F}$, the state transition matrix $\varnothing$, and the observation matrix $H$. But for nonlinear systems, these are the functions of the state, and change consequently with every time step, so they cannot be pre-computed.

\section{EKF PREDICTION}

In the nonlinear case the prediction can be calculated as in linear case, but it should be noted that, the use of matrices are not constant like in linear case, but depend on time step;

$$
P^{-}\left(t_{i}\right)=\phi_{t_{i-1}}^{t_{i}} \cdot P\left(t_{i-1}\right) \cdot\left(\phi_{t_{i-1}}^{t_{i}}\right)^{T}+\int_{t_{i-1}}^{t_{i}} Q(t) d t_{\ldots \ldots .}(
$$

\section{EKF CORRECTION}

Like the differential equation in the prediction step, the corresponding nonlinear observation equations are linearized with the Taylor series about the predicted state $x^{-}\left(t_{i}\right)$, and higher order terms are neglected.

Thus the approximation observation matrix is

$$
H\left(t_{i}\right)=\frac{\partial h(x)}{\partial x} \mathbb{I}_{x=x^{\prime}}\left(t_{\mathrm{i}}\right)
$$

In this case the predicted measurement $\mathbb{P}^{-}\left(t_{i}\right)$ for calculating the measurement residual is $\mathbb{I}^{-}\left(t_{i}\right)=h\left(x^{-}\left(t_{i}\right)\right)$, the same formula is used to calculate the corrected state, and its covariance matrix like in the linear, but with time dependent matrices[11,12].

$$
P^{+}\left(t_{i}\right)=\left(I-K\left(t_{i}\right) H\left(t_{i}\right)\right) P^{-(}\left(t_{i}\right)
$$

$$
K\left(t_{i}\right)=P^{-} H\left(t_{i}\right)^{T}\left(H\left(t_{i}\right) P^{-} H\left(t_{i}\right)^{T}+R\left(t_{i}\right)\right)^{-1}
$$

\section{RESULT}

The KF was tested for latitude, longitude of true trajectory, and velocity north of true trajectory with INS, and INS/GPS, while EKF was tested for position, and velocity of true trajectory. The result was shown in figure 1 through figure 5 . Figure 6 shows the behavior of EKF when it is tested on sinus wave.

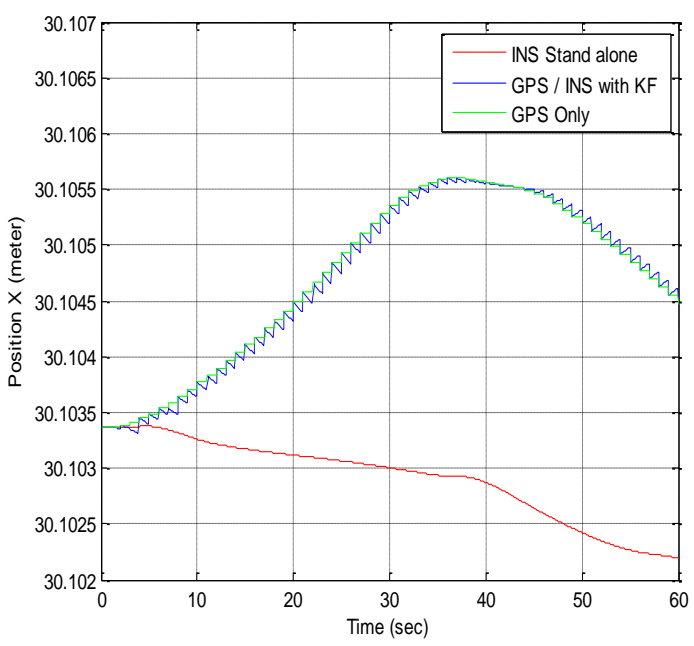

Fig 1 : Comparison between Latitude of true trajectory by GPS and trajectory by GPS/INS with KF

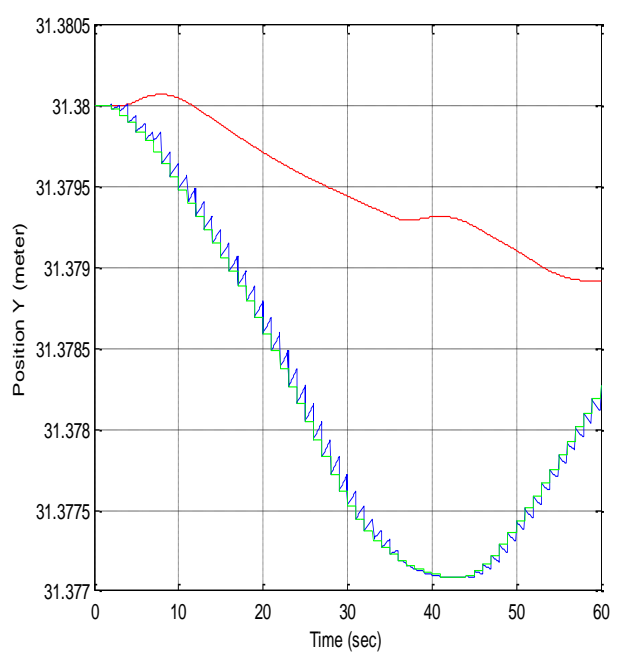

Fig 2 : Comparison between Longitudes of true trajectory by GPS and trajectory by GPS/INS with KF

With 


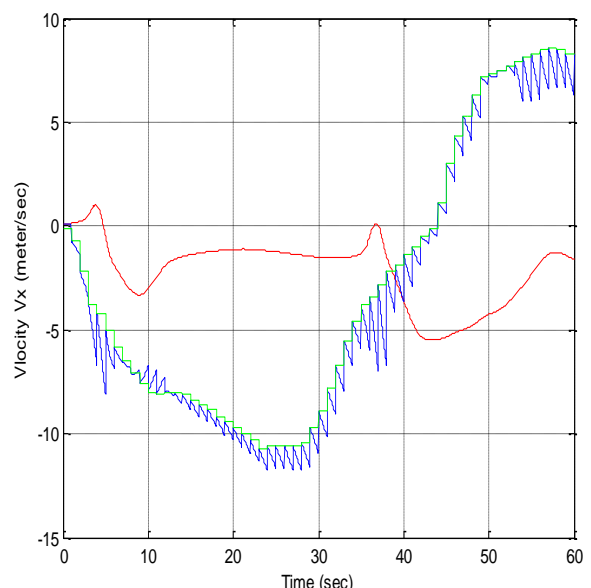

Fig 3: Comparison between Velocity North of true trajectory by GPS and trajectory by GPS/INS with KF

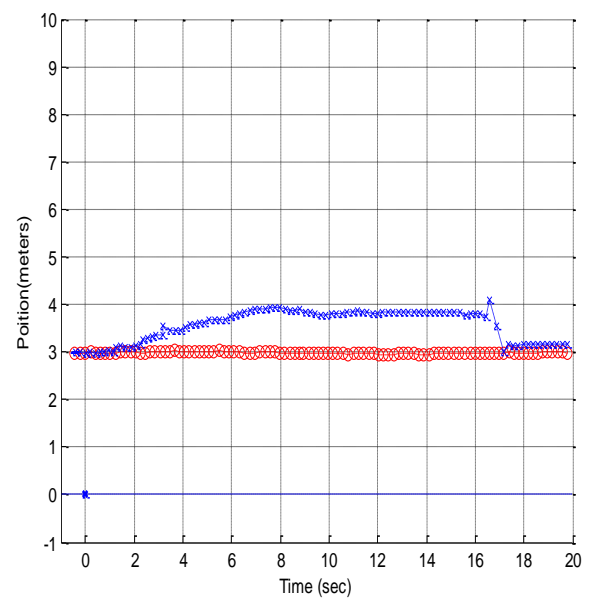

Fig 4: Position of true trajectory by GPS and trajectory by INS with EKF.

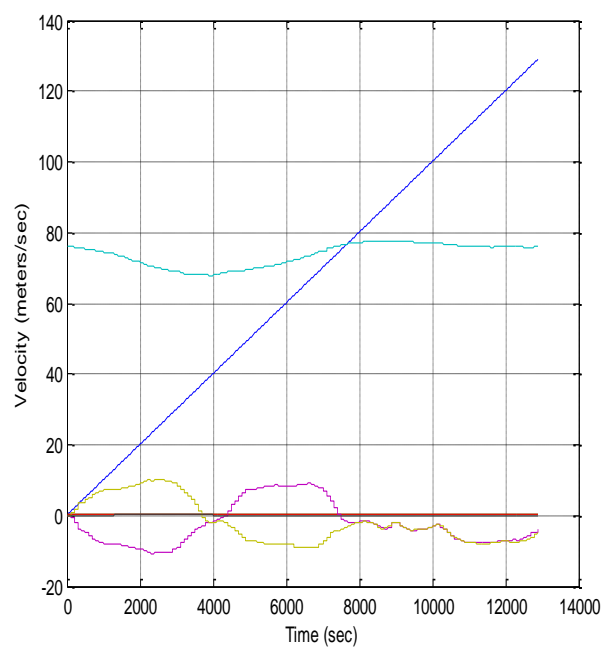

Fig 5: Velocity of true trajectory by GPS and trajectory by GPS/INS with EKF

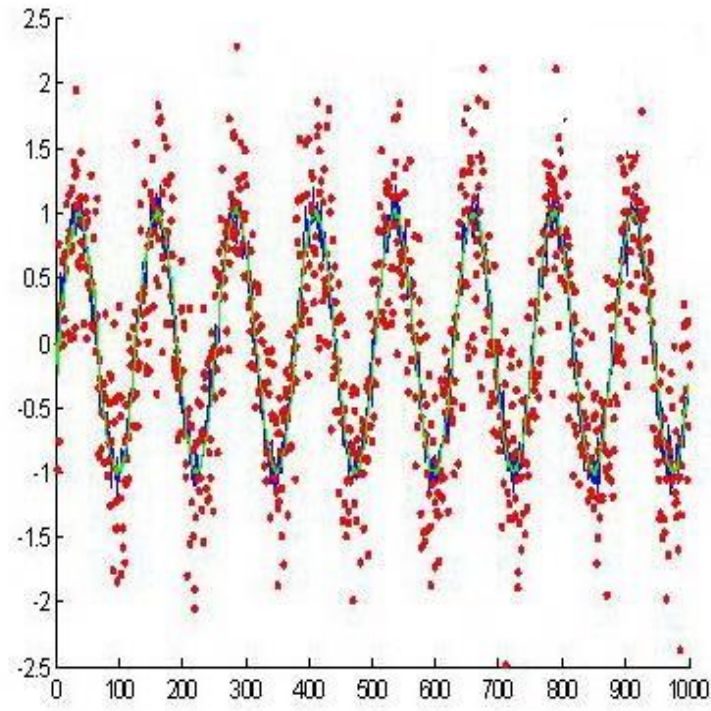

Fig 6: EKF tested on sinus wave

\section{CONCLUSION}

In this paper, compares between Kalman and extended Kalman filtering of aircraft tracking for orientation data represented with quaternions. The results indicate that, although the KF and EKF have roughly the same accuracy, the $\mathrm{KF}$ is better choice for liner system applications, and the quasi-linear nature of the quaternion dynamics makes the EKF a better choice for the task of improving tracking of noisy quaternion signals in virtual reality applications.

\section{REFERENCES}

[1] B. Hoffmann-Wellenhof, H. Lichtenegger and J. Collins, 1997. GPS Theory and Practice, Springer-Verlag Vienna.

[2] Mohinder S. Grewal, Angus P. Andrews, 2001. Kalman Filtering Theory and Practice Using Matlab,John Wiley \& Sons Inc 2001.

[3] Sorenson, Harold W. ,1995. Kalman filtering theory and application ,New York

[4] Rachel Kleinbauer, Universität Stuttgart, Helsinki, Nov. 2004, Kalman Filtering Implementation with Matlab.

[5] D. Bouvet and G. Garcia. Improving the Accuracy of Dynamic Localization Systems using RTK GPS by Identifying the GPS Latency, IEEE.

[6] Qi. Honghui and J. B. Moore. Direct Kalman Filtering Approach for GPS/INS Integration. IEEE Transactions on Aerospace and Electronic Systems, 38(2):687-693, 2002.

[7] Shaik Sami, P.Padmaja, International Journal of Science and Research (IJSR), India Online ISSN: 2319-7064, Vol. 2 Issue 5, May 2013, Speech Enhancement Using Fast Adaptive Kalman Filtering Algorithm Along With Weighting Filter

[8] R. Sharaf, A. Noureldin, A. Osman, and N. El-Sheimy. Online INS/GPS Integration with A Radial Basis Function Neural Network. IEEE Aerospace and Electronic Systems Magazine, 20(3):8-14, 2005. 
[9] M. Grewal, L. Weill, and A. Andrews, Global Positioning Systems, Inertial Navigation and Integration, 2ed, Hoboken, NJ, Wiley, 2007.

[10] L. Zhao, W. Ochieng, M. Quddus, and R. Noland, An extended Kalman filter algorithm for integrating GPS and low cost dead reckoning system data for vehicle performance and emission monitoring, J. Navig, vol. 56, no. 2, pp. 257-275, May 2003.
[11] Pamadi, B. N., Performance, Stability, Dynamics and Control of Airplanes, AIAA Education Series, Virginia, 1998.

[12] S. Rezaei and R. Sengupta, Kalman filter-based integration of DGPS and vehicle sensors for localization, IEEE Trans. Control Syst. Technol., vol. 15, no. 6, pp. 1080-1088, Nov. 2007. 